\title{
Tests génétiques en accès libre sur Internet
}

$>$ Un phénomène nouveau reliant les avancées de la biotechnologie aux technologies de l'information et de la communication semble s'être produit sans qu'on en ait anticipé la nature et l'ampleur. II s'agit de la mise à disposition, sur Internet, d'une génomique dite personnalisée. Les usagers peuvent acheter directement auprès de diverses compagnies une offre combinée de tests génétiques pour de multiples maladies, associant parfois des informations relatives à l'ancestralité, sans qu'il soit nécessairement requis de prescription médicale et sans que l'usager soit systématiquement assisté par un professionnel de santé pour l'interprétation des résultats. Quels types de stratégies commerciales utilisent les entreprises offrant des tests génétiques en libre accès sur Internet et sur quelles catégories d'attentes sociales jouent-elles parallèlement? Par une analyse tant quantitative que qualitative des sites Internet qui proposent ces tests, on constate que ces compagnies visent un triple marché : celui du healthism (santéisme) qui a hissé la santé et l'hygiène au panthéon des valeurs sociales; celui des revendications contemporaines des usagers des systèmes de santé à devenir de véritables acteurs des décisions de santé ; et enfin celui du lien (bio-)social. Ces trois stratégies commerciales recouvrent différents enjeux éthiques et sociétaux qu'il convient d'analyser. <

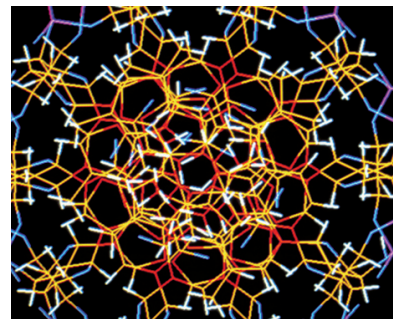

et est aussi couramment utilisée aujourd'hui pour parler de la vente directe de tests génétiques via Internet. L'apparition de ce nouveau marché suscite de nombreuses interrogations [1]. Pour les comités d'éthique et les institutions en charge des questions de santé, il s'agit d'une situation nouvelle: la législation tente d'encadrer l'utilisation des tests génétiques en général (Encadré I), mais elle est dépassée par un marché électronique et globalisé des tests qui échappe à de nombreuses régulations nationales et régionales [2]. Dans ce contexte, différentes autorités tentent de se faire entendre pour demander la mise en place de nouvelles régulations au niveau national ${ }^{1}$ ou au niveau européen (Encadré 2).

${ }^{1}$ On peut par exemple citer les travaux du Comité consultatif national d'éthique qui avait, dès 2004, commencé à prendre en compte la situation nouvelle créée par l'apparition des «autotests» (voir l'avis ${ }^{\circ} 86$ de novembre 2004), ceux de l'Agence de la biomédecine (voir le bilan d'application de la loi de bioéthique d'octobre 2008), ceux de l'Office parlementaire d'évaluation des choix scientifiques et technologiques (voir le rapport daté de novembre 2008 sur l'évaluation de l'application de la loi du 6 août 2004 relative à la bioéthique), ou encore le rapport final des États généraux de la bioéthique (Mission parlementaire, janvier 2010). 


\section{Exemple d'une législation nationale encadrant la pratique des tests génétiques : les textes applicables en France}

Domaine d'application des tests génétiques

Article 16-10, code civil alinéa 1. L'examen des caractéristiques génétiques d'une personne ne peut être entrepris qu'à des fins médicales ou de recherche scientifique.

Article 16-13, code civil. Nul ne peut faire l'objet de discriminations en raison de ses caractéristiques génétiques.

Modalités de réalisation et annonce des résultats

Article 16-10 code civil alinéa 2. Le consentement exprès de la personne doit être recueilli par écrit préalablement à la réalisation de l'examen, après qu'elle a été dûment informée de sa nature et de sa finalité. Le consentement mentionne la finalité de l'examen. II est révocable sans forme et à tout moment.

Article L1131-1 code de la santé publique alinéa 2. Toutefois, lorsqu'il est impossible de recueillir le consentement de cette personne ou, le cas échéant, de consulter la personne de confiance mentionnée à l'article L. 1111-6, la famille ou, à défaut, un de ses proches, l'examen ou l'identification peut être entrepris à des fins médicales, dans l'intérêt de la personne.

Les dispositions règlementaires

Articles R1131-4 et suivants, code de la santé publique.
Ces articles organisent les conditions de réalisation du test en spécifiant les modalités de l'information donnée au patient, de prescription du test, ainsi que celles de l'annonce des résultats.

Le code de la santé publique prévoit en outre les conditions à remplir pour les professionnels (agrément) et pour les laboratoires (autorisation) afin de réaliser un test génétique.

\section{Les propositions}

Dans le cadre de la révision des lois de bioéthique de 2004, la question de l'encadrement des tests génétiques en accès libre a été évoquée par l'Office parlementaire des choix scientifiques et technologiques («L'évaluation de l'application de la loi du 6 août 2004 relative à la bioéthique », 20 novembre 2008), l’Agence de la biomédecine ( $«$ Le bilan d'application de la loi de bioéthique du 6 août $2004 », 2008$ ) et la mission d'information sur la révision des lois bioéthiques (Rapport d'information $n^{\circ} 2235$ déposé le 20 janvier 2010). Ces instances proposent d'une part que soit inscrite dans la loi l'interdiction pour les particuliers de se prévaloir des résultats de ces tests en France, et d'autre part que l'Agence de la biomédecine soit chargée d'une veille sur les sites proposant ces tests afin d'en assurer la qualité et la validité.

\section{Quelques exemples de textes européens prenant position sur l'offre de tests génétiques en libre accès}

Le protocole du Conseil de l'Europe

Protocole additionnel à la Convention sur les droits de l'Homme et la biomédecine relatif aux tests génétiques à des fins médicales, Strasbourg, 27.11.2008 (http://conventions.coe.int/Treaty/fr/ Treaties/html/203.htm).

Ce protocole rappelle les grandes conditions de validité de réalisation des tests génétiques à visée médicale concernant l'information, le consentement écrit, la place de la famille et la réalisation des tests sur des personnes décédées. Les tests ne peuvent être accessibles que sur prescription médicale. L'utilité clinique des tests doit être démontrée.
Cet instrument juridique a l'originalité de faire des propositions pour la mise en œuvre de programmes de dépistage génétique à des fins médicales. Parlement Européen

Science and Technology Options Assessment (STOA)

Direct To Consumer Genetic Testing Study 2008 http://www.europarl. europa.eu/stoa/publications/studies/stoa32and39_en.pdf

Ce rapport fait le point sur les discussions menées par les experts et les autorités publiques sur la question des tests génétiques en accès libre sur Internet. À partir d'une analyse du développement du marché et des thèses en présence, ce rapport identifie les besoins et possibles solutions pour les politiques publiques.
On observe aussi diverses réactions des professionnels de la médecine et de la génétique qui s'expriment soit individuellement soit par le biais de leurs associations professionnelles ${ }^{2}$, pour demander des réponses appropriées à cette nouvelle offre [3]. Le débat professionnel se heurte à plusieurs questions: la validité scientifique de tels tests génétiques [4], leur utilité clinique $[5,6]$, et enfin le caractère direct de cette offre qui ne s'accompagne ni d'une prescription ni d'un accompagnement médical dans l'interprétation des résultats [7, 8]. L'apparition d'une offre de participation à la recherche en génomique (dénommée direct research par ses promoteurs) pour un prix promotionnel va très probablement intensifier ce débat puisque celle-ci tend à mettre à mal le modèle d'une recherche conduite sous contrôle académique et universitaire.

${ }^{2}$ À titre d'exemple, voir les textes publiés par la Société européenne de génétique humaine : https://www. eshg.org.
L'abondance de ces réactions se comprend face à un phénomène devenu incontournable et un véritable succès commercial, du moins vu de l'extérieur ${ }^{3}$. Le nombre d'entreprises offrant des tests en accès direct est en augmentation (42 sites recensés en 2009 par nos soins (Encadré 3) contre 38 en 2008 recensés par Hennin et al. [9]) alors que selon ces auteurs, ce marché n'a débuté qu'au début des années 2000. Quelles raisons expliquent cet apparent succès? Quels types de stratégies ou de pratiques commerciales utilisent ces entreprises pour

${ }^{3}$ Nous ne disposons pas réellement, à ce stade de nos investigations, de données sur le nombre de personnes ayant acheté via Internet des tests génétiques à dimension médicale, ce type de données n'étant pas communiqué publiquement par les entreprises. Cependant, à titre d'exemple, nous pouvons rapporter ici les déclarations du chargé de communication d'une des entreprises leaders de ce marché, au cours d'un entretien: sa compagnie, fondée en 2006, avait comptabilisé un peu plus de 10000 clients pour les huit premiers mois de l'année 2009. 


\section{Liste des 42 sites internet recensés et étudiés et des modalités d'accès aux tests proposés}

23 and Me, BioResolve, DeCodeMe, Navigenics, Labgenetics, Athenadiagnostics, Agendia, Geneessence, Carolyn Katzin's The DNA Diet, Great Lakes Genetics, Consumer Genetics, CyGene Direct, Dermagenetics, DNA dimensions, DNA direct, Dna Traits, Eastern Biotech Lifesciences, Enterolab, Genova Diagnostics, GeneDX, Genelex, GeneLink Biosciences, Genetic Technologies, Genzymegenetics, Gracefulearth, Hairdx, Healthcheck, Hivgene, Psynomics, IMMD (Institute of medical molecular diagnostics of $\mathrm{GmbH}$ ), Inneova, Kimballgenetics, Mygenome, Myriad, New Hope Medical, G-nostics, Genetic Health, Interleukin Genetics, Salugen (Genotrim), Holistic Health consultants, Knome, Atlas Genes.
Sur ces 42 sites, 18 proposent de réaliser un ou des tests, sur simple demande de l'usager, sans qu'il y ait d'entretien préliminaire requis; 10 , après avoir pris contact avec un conseiller génétique travaillant directement pour le site, ou avec un conseiller ou un médecin extérieur dont les coordonnées sont communiquées à l'internaute; 4 , après avoir obtenu la prescription d'un médecin, sans préciser de coordonnées pour en trouver un susceptible de la faire ; 9 ne précisent pas vraiment les modalités d'obtention; 1 ne propose pas de pratiquer directement des tests mais simplement de les interpréter. convaincre les usagers de recourir à leurs services? Sur quelles attentes sociales jouent-elles parallèlement? Une première réponse à ces questions vient d'une analyse fine et quantifiée des tests mis en vente sur Internet qui devrait permettre d'appréhender assez facilement le type de marché visé. Une seconde réponse, prolongeant la première, vient non plus tant de l'analyse du fond que de la forme de l'offre, en particulier les procédés aussi bien graphiques qu'argumentatifs qu'elle utilise. L'identification de ces ressorts commerciaux révèle des enjeux éthiques et sociétaux sur lesquels nous reviendrons en conclusion.

\section{Des maladies les plus communes aux plus fréquentes des maladies rares}

Les entreprises offrent des tests de susceptibilité et de prédisposition d'une extrême diversité : pathologies multifactorielles des plus communes aux plus rares, maladies monogéniques, maladies mentales, réactions métaboliques, ou encore ancestralité des personnes. Enfin, certaines entreprises proposent des tests liés aux performances physiques et intellectuelles, aux troubles du comportement (impulsivité, inattention, etc.), et à l'intelligence, ce qui est fort problématique à divers points de vue ${ }^{4}$. Si l'extraction et le transport de l'échantillon à analyser s'opèrent de manière relativement homogène (les usagers renvoient par la poste un tube dans lequel ils ont déposé un prélèvement de cellules épithéliales de la cavité buccale), les modes de délivrance des résultats des tests sont très divers : certains sites semblent autoriser leurs services aux mineurs $[10,11]$, d'autres s'y opposent; certains préconisent une consultation avec un médecin ou un conseiller génétique avant ou après le test, d'autres non. De même, les coûts varient en fonction des sites et tendent à baisser (entre 200 euros et 2000 euros [12] en 2007, à partir de 60 euros aujourd'hui).

\footnotetext{
${ }^{4}$ D'une part, nous pouvons nous demander si les facteurs environnementaux, tels que les processus de socialisation par exemple, dont on sait le rôle déterminant dans le développement de ces traits, sont pris en compte. D'autre part, en faisant une telle proposition de test, les entreprises semblent ignorer le fait que la définition empirique de ces traits suppose des visions - socialement et culturellement situées - du monde, de ses catégories et hiérarchies. Enfin, la pratique de tels tests risque d'aboutir à une «essentialisation » de ces catégories sociales et culturelles, masquant du même coup le point de vue, et éventuellement les intérêts, de leurs producteurs et utilisateurs.
}

Nous avons recensé 42 sites proposant plus de 700 tests différents, et les plus couramment offerts sont présentés dans le Tableau I. Nous avons choisi de ne retenir dans ce tableau que ceux classés parmi les 10 premiers du palmarès. Les tests les plus courants concernent les maladies communes et multifactorielles (maladies cardiovasculaires au sens large, cancers, obésité, diabète, etc.). Si nous reprenons ici la terminologie d'0mran [13], les entreprises se situent tout à fait en phase avec l'époque (actuelle) où les maladies dégénératives et celles induites par l'activité humaine succèdent, dans un processus dit de «transition épidémiologique», à une époque où la mortalité était principalement due à des maladies infectieuses et parasitaires. En définissant comme prioritaires ces pathologies contemporaines, les entreprises se font l'écho des préoccupations des politiques publiques et des citoyens, et anticipent des perspectives de développement. Parallèlement, figurent dans ce top 10 des tests les plus fréquemment proposés pour la fibrose kystique (mucoviscidose), I'hémochromatose et la maladie de Tay-Sachs, soit les plus fréquentes des maladies rares et monogéniques. II y a là un opportunisme certain.

Enfin, on note, en bonne position dans ce palmarès, deux types de tests qui n'ont pas directement pour but de décrire une susceptibilité génétique au développement d'une pathologie particulière: il s'agit des tests relatifs au métabolisme de molécules médicamenteuses et de nutriments et des tests ayant trait aux performances physiques ou présentés comme tels. Ces derniers recherchent des marqueurs génétiques prédisposant aux performances athlétiques et musculaires, ou simplement à la «forme physique ». Si la proposition d'une pharmacogénomique en accès direct prolonge des pratiques déjà existantes dans un cadre médical spécialisé (par exemple en cancérologie lors de la prescription de chimiothérapies), les tests génétiques appliqués au 


\begin{tabular}{|c|c|c|c|}
\hline & $\begin{array}{l}\text { Nombre } \\
\text { de sites }{ }^{1}\end{array}$ & $\begin{array}{c}\% \text { de sites } \\
\text { proposant le test }^{2}\end{array}$ & Rang \\
\hline $\begin{array}{l}\text { Infarctus/maladies } \\
\text { cardiovasculaires }\end{array}$ & 24 & 57,1 & 1 \\
\hline Cancer du sein & 16 & 38,1 & 2 \\
\hline Pharmacogénomique & 14 & 33,3 & 3 \\
\hline Maladie d'Alzheimer & 13 & 31 & 4 \\
\hline Cancer du poumon & 13 & 31 & 4 \\
\hline Diabète de type 2 & 12 & 28,6 & 4 \\
\hline $\begin{array}{l}\text { Fibrose } \\
\text { kystique/mucoviscidose }\end{array}$ & 12 & 28,6 & 5 \\
\hline Cancer de l'estomac & 11 & 26,1 & 6 \\
\hline Hémochromatose & 11 & 26,1 & 6 \\
\hline Obésité & 11 & 26,1 & 6 \\
\hline Ostéoporose & 11 & 26,1 & 6 \\
\hline Cancer de l'ovaire & 11 & 26,1 & 6 \\
\hline Cancer de la prostate & 11 & 26,1 & 6 \\
\hline Accident vasculaire cérébral & 11 & 26,1 & 6 \\
\hline Cancer du côlon & 10 & 23,8 & 7 \\
\hline Cancer colorectal & 10 & 23,8 & 7 \\
\hline Thrombophilie & 10 & 23,8 & 7 \\
\hline Polyarthrite rhumatoïde & 10 & 23,8 & 7 \\
\hline Asthme & 9 & 21,4 & 8 \\
\hline Maladie cœliaque & 9 & 21,4 & 8 \\
\hline $\begin{array}{l}\text { Performance } \\
\text { musculaire/athlétique }\end{array}$ & 8 & 19 & 9 \\
\hline Maladies auto-immunes & 7 & 16,7 & 10 \\
\hline Calculs biliaires & 7 & 16,7 & 10 \\
\hline Sclérose en plaques & 7 & 16,7 & 10 \\
\hline $\begin{array}{l}\text { Test génétique } \\
\text { nutritionnel/nutrigénomique }\end{array}$ & 7 & 16,7 & 10 \\
\hline Cancer de la peau & 7 & 16,7 & 10 \\
\hline Maladie de Tay-Sachs & 7 & 16,7 & 10 \\
\hline Diabète de type 1 & 7 & 16,7 & 10 \\
\hline
\end{tabular}

Tableau I. Tests génétiques les plus fréquemment proposés par les 42 sites étudiés. ${ }^{1}$ Sites offrant le ou les tests recensés. ${ }^{2} \%$ des sites proposant chaque test. métabolisme des aliments et aux performances physiques sont originaux. On peut néanmoins s'interroger sur le type de demandes sociales qu'ils cherchent à capter et à développer. Si l'on peut y voir une «généticisation » relativement inédite de certains domaines de la vie sociale (en l'occurrence ici l'alimentation et la performance), ils semblent aussi accompagner un mouvement, déjà largement engagé, de médicalisation sociale - analysé entre autres par Aïach [14, 15] - dans lequel la compétence biomédicale s'est étendue à de multiples dimensions de la vie individuelle et collective.

\section{Un marché du « santéisme ${ }^{5}$ »}

En deçà de cette dynamique de médicalisation, les tests proposés par les sites s'inscrivent dans une évolution culturelle, politique et idéologique de nos sociétés contemporaines qui place la santé au panthéon des valeurs et s'accompagne d'une nouvelle vision du monde fondée sur la promesse d'une longévité physique et intellectuelle pour qui saurait se conduire selon des principes hygiénistes [16]. Ce mouvement que l'on peut nommer healthism ou «santéisme » à la suite d'Aïach a donné lieu, selon ce dernier, à une transformation importante des habitudes: les individus se sont mis à déclarer plus de symptômes morbides, ont prêté plus d'attention à de potentiels troubles de santé et adopté de multiples comportements préventifs.

Si par leur positionnement sur le créneau des «maladies de civilisation » les sites s'assurent une place au cœur des préoccupations contemporaines de santé, leur marketing accentue ce souci sanitaire par une exploitation habile des tendances du santéisme et des craintes qui en découlent. Les slogans affichés sur les pages d'accueil des sites illustrent la tonalité de cette communication qui invite à se soumettre sans plus tarder à des tests afin d'accroître son espérance de vie: on lit par exemple sur un site: Confront risk early, prolong your health; sur un second: Genetic testing for a healthier future. Pour déclencher l'acte d'achat, d'autres sites jouent en outre sur le registre de l'anxiété, dramatisant la description des maladies. Sur sa page d'accueil, un site lance l'avertissement suivant: Knowing your DNA could save your life!; un second, spécialisé dans la nutrigénomique du cerveau, annonce: It runs in the family.

Le rapport sur le risque génétique proposé une fois le test réalisé renvoie de même au mouvement santéiste en ce qu'il attire principalement l'attention sur des

${ }^{5}$ Le «santéisme», d'après le terme healthism, utilisé aux États-Unis, est un mouvement qui place la santé en tête des valeurs et préoccupations sociales. 
écarts de prévalence de pathologies ou de traits entre le groupe génotypique de l'usager et la population générale. Un risque relatif pour chacun des traits et des pathologies testés (présenté selon trois catégories: risque faible, standard ou élevé) est communiqué à la personne. Le test peut ainsi classer dans la catégorie des risques élevés une maladie dont la prévalence est faible, entraînant une attention démesurée. La question de l'appréciation des modifications du risque se pose. On peut par ailleurs observer que la présentation des résultats est à la fois partielle et partiale (au moins dans les démonstrations figurant sur les sites) puisque seules certaines mesures statistiques sont présentées, sans indication de précision (intervalle de confiance) et vraisemblablement selon des hypothèses hautes quant à la distribution des variants testés en population générale ${ }^{6}$. Pour illustrer la gamme d'interprétations possibles, nous présentons dans l'Encadré 4 différentes manières d'interpréter un même risque ${ }^{7}$. On observe que le recours à certaines statistiques plutôt qu'à d'autres est fait de manière à valoriser les interprétations les plus frappantes ou les plus alarmistes. En outre, la présentation des risques s'appuie généralement sur une estimation haute de la prévalence et des intervalles de confiance associés au risque de pathologie. Le sentiment d'anxiété sanitaire sur lequel joue implicitement le marketing en est d'autant plus renforcé. Dans ce contexte global de saisie des risques, la logique de construction du normal et du pathologique par comparaison systématique à la moyenne [17] est renforcée, avec tous les travers que cela peut comporter (l'écart à la moyenne devient en soi problématique, suppose anormalité et donc risque). (eci surviendrait au détriment d'une autre appréhension des risques, plus proche de l'individu et de ses composantes biologiques, sociales et environnementales, qui reposerait sur une appréciation de l'individu dans son contexte de vie.

\section{Un marché de la « biopolitique individuée ${ }^{8}$ » et de la curiosité pour la génomique}

Après le santéisme, le second ressort sociétal de ce marché des tests génétiques est celui des revendications contemporaines des usagers des systèmes de santé qui se veulent de véritables acteurs/auteurs des décisions de santé qui les concernent. Le marketing s'appuie en effet sur des thématiques largement répandues dans l'opinion publique dont l'affirmation de rapports alternatifs dans la relation patient/médecin (droit de savoir, empowerment du patient, appropriation et partage de la connaissance médicale, etc.), en

\footnotetext{
${ }^{6}$ Ces variants sont établis sur les bases du paradigme contesté de la recherche en génétique multifactorielle qui consiste à attribuer la majeure partie de la susceptibilité génétique à des variants communs: common disease common variant. Cette hypothèse permettait de justifier le recours aux études de type génome entier (Genome wide association study, GWAS). Bien qu'ayant permis d'impliquer des dizaines de gènes dans la plupart des maladies génétiques multifactorielles, ces études ont un succès indéniable, mais qui reste partiel pour au moins deux raisons : (1) le variant commun peut n'être qu'une approximation d'un autre plus rare et plus associé (synthetic association); (2) l'ensemble des gènes découverts n'explique qu'une part modeste de la variabilité observée.

${ }^{7}$ L'encadré 4 présente l'analyse qui pourrait être faite des données. Ce que les compagnies pourvoyeuses de tests sur Internet ne font pas.

${ }^{8}$ Dans une « biopolitique individuée » l'État, déléguant à l'individu la gestion rationnelle du donné corporel, ne s'occuperait plus que de la «surveillance des autosurveillances» (repris de [19]).
}

mettant en avant le caractère direct de l'offre. Un site clame par exemple: Your health, your right to know ; un autre: Personal control over private genetic information; et un troisième propose: Empowering individuals with the predictive power of DNA. D'autres sites insistent plutôt sur l'offre aux usagers-individus d'une biotechnologie individualisée ou personnalisée, inexistante jusqu'à présent, et jouent sur la nouveauté de l'offre et la curiosité du public vis-à-vis des avancées de la génomique: Genome sequencing for individuals, annonce l'un; Genetics just got personal, dit un autre.

Tout en proposant une généticisation inédite, les entreprises mettent en avant les capacités d'innovation et de libre détermination que les personnes peuvent retirer de l'usage de leurs services. L'usager peut ainsi mieux adapter ses comportements en fonction des résultats des tests: Make better lifestyle choices ou Prepare for serious diseases prône cette philosophie. À une vision de la génétique souvent dominée par la figure du déterminisme biologique et du fatum est substituée une figure de l'indétermination et de la maîtrise individuelle de la destinée sanitaire. There's DNA. And then there's what you do with it. [Our] genetic test gives you insight into your personal health, proclame un des sites.

Certains invitent l'usager à devenir acteur de la recherche en génomique au lieu de rester un simple consommateur de service, cela pour un prix promotionnel (25 dollars le test). Plus que le prix attractif proposé, la communication met en avant un nouveau type de recherche médicale (Get involved in a new way of doing research), réalisé au plus près de l'individu et de ses préoccupations (Direct research by participating in studies of conditions and traits you care about). Là encore, la présentation insiste sur la rupture et sur l'émergence d'une nouvelle manière de prendre en compte les usagers, rompant avec les modes de recherche plus traditionnels des institutions médicales où le participant n'a guère que le statut de pourvoyeur de données [18]. La mise en valeur de l'individu et de son émancipation face à l'ignorance et aux tutelles, le corps médical dans ses comportements traditionnels ou la technocratie, est une des clefs de voûte du marketing. Les compagnies pourvoyeuses de tests tentent ainsi de capter et de prolonger un mouvement social qui va vers une gestion de la vie de plus en plus déléguée aux individus (une «biopolitique individuée») et de moins en moins dirigée par les États et les corps professionnels de la médecine, mouvement observé et commenté par différents auteurs dans d'autres contextes $[19,20]$. 


\section{Différentes manières d'interpréter un même risque génétique}

\section{Scénario pour 100000 individus}

- Maladie multifactorielle de prévalence $1 \%$ ( 1 malade pour 1000 individus).

- Marqueur SNP dont l'allèle mineur a une fréquence de $20 \%$ environ, $36 \%$ des individus en sont porteurs.

- L'association entre le portage de cet allèle et la maladie se traduit par un odds ratio de 2,2.

\begin{tabular}{|c|c|c|c|}
\hline & Malades & Non atteints & \\
\hline $\begin{array}{l}\text { Porteurs du risque génétique } \\
\text { (test génétique +) }\end{array}$ & $\begin{array}{l}\mathbf{5 5} \\
\text { Malades et porteurs du risque } \\
\text { génétique (vrais positifs) }\end{array}$ & $\begin{array}{l}\qquad 35725 \\
\text { Non atteints mais porteurs du risque } \\
\text { génétique (faux positifs) }\end{array}$ & $35,8 \%$ \\
\hline $\begin{array}{c}\text { Non-porteurs du risque } \\
\text { génétique (test génétique -) }\end{array}$ & $\begin{array}{l}\mathbf{4 5} \\
\text { Malades non-porteurs du risque } \\
\text { génétique (faux négatifs) }\end{array}$ & $\begin{array}{l}\quad 64175 \\
\text { Non atteints et non-porteurs du risque } \\
\text { génétique (vrais négatifs) }\end{array}$ & $64,2 \%$ \\
\hline Total $=100000$ & 100 & 99900 & $100 \%$ \\
\hline
\end{tabular}

Remarque : ce scénario est compatible avec rs31353888 (marqueur de HLA-DRB1*15:01) pour une maladie multifactorielle à composante génétique comme la sclérose en plaques (SEP) [24]. La prévalence retenue de la SEP est de l'ordre de grandeur de celle généralement admise en France, probablement sous-estimée [25].

\section{Statistiques}

Sont ici présentés 6 indicateurs statistiques de la force de l'association entre le marqueur génétique et la présence de la maladie par rapport à des individus non atteints. Leur emploi fait un large consensus au-delà du domaine de l'épidémiologie génétique.

$\begin{array}{lll}\text { Différence de risque : } & 0,84 \% & {[0,38 \% ; 1,29 \%]} \\ \text { Risque relatif : } & 2,19 & {[1,48 ; 3,25]} \\ \text { Sensibilité statistique : } & 55,0 \% & {[44,7 \% ; 65 \%]} \\ \text { Spécificité statistique : } & 35,8 \% & {[35,5 \% ; 36,1 \%]} \\ & & (\text { spécificité statistique : } \\ & 64,2 \%[63,9 \% ; 64,5 \%]) \\ & & \end{array}$

\section{Interprétations}

Chaque indicateur statistique est en tant que tel. Les divers modèles génétiques d'associations statistiques possibles entre le polymorphisme génétique et la maladie ne sont pas discutés. Nous donnons des indications de calculs.

- Interprétation dite du « risque relatif » : il y a 2,19 fois plus de chances d'observer la maladie chez les porteurs de l'allèle dit à risque que les chez les non-porteurs.

Le risque relatif est calculé en effectuant le ratio entre le risque chez les porteurs de l'allèle et le risque chez les non-porteurs, et s'interprète donc comme le facteur multiplicatif associé à l'information génétique concernant le risque.

- Interprétation dite de la « différence de risque »: il y a 0,84 chance sur 1000 en plus d'observer la maladie chez les porteurs de l'allèle dit à risque comparé aux non-porteurs. La différence de risque est calculée en effectuant la différence entre le risque chez les porteurs de l'allèle et le risque chez les nonporteurs et s'interprète donc comme le facteur additif associé à l'information génétique concernant le risque.
- Interprétation dite de la « sensibilité statistique » : $55 \%$ des personnes atteintes par la maladie sont porteuses de l'allèle dit à risque.

La sensibilité est calculée chez les atteints, et indique la proportion de patients porteurs de l'allèle dit à risque.

Contrairement aux maladies monogéniques, il permet de se rendre compte que l'allèle dit à risque n'est ni nécessaire ni suffisant pour engendrer la maladie.

- Interprétation dite de la « spécificité statistique 》 : $35,8 \%$ des personnes non atteintes par la maladie sont porteuses de l'allèle dit à risque.

La probabilité complémentaire de la spécificité (« 1-spécificité ») est calculée chez les non atteints. Elle indique la proportion de porteurs de l'allèle dit à risque. Elle souligne combien le variant dont il est question est fréquent dans la population générale.

- Interprétation dite de la « valeur prédictive positive 》: les personnes porteuses de l'allèle dit à risque ont $0,15 \%$ de chance d'être atteintes de la maladie.

La valeur prédictive positive est calculée chez les personnes porteuses de l'allèle dit à risque. Comparé à la fréquence de la maladie en population générale, elle mesure l'information du test chez les porteurs de l'allèle à risque. Il existe également une valeur prédictive négative pour les non-porteurs.

De plus, chacune des statistiques ci-dessus pourrait être présentée selon une hypothèse basse en utilisant la borne inférieure de l'intervalle de confiance (noté entre crochets) associée à l'estimation du paramètre ou selon une hypothèse haute en utilisant sa borne supérieure. L'intervalle de confiance permet de rendre compte de l'imprécision liée aux fluctuations d'échantillonnage des valeurs qui sont estimées à partir d'un échantillon, néanmoins, leur interprétation peut considérablement varier en fonction de cette imprécision. 
Join the community : un marché du lien (bio-)social et de la démocratie médicale

Plusieurs des sites internet que nous avons étudiés développent des blogs et forums liés à leurs pages, et/ou renvoient vers des blogs hébergés sur d'autres sites. Des pictogrammes ergonomiques symbolisant le partage et le groupe sont régulièrement utilisés pour servir de passerelle vers ces espaces de discussion numérique. Le principe de ces espaces est triple. Ils permettent à l'usager de dialoguer directement avec les personnes qui administrent les sites, de discuter avec d'autres usagers - ces espaces renvoient ainsi à la formation d'une démocratie médicale et scientifique autour de la génomique - mais aussi, sur certains sites, de se faire des amis par le biais d'un partage d'informations génétiques personnelles. Un des sites propose ainsi l'onglet Search for and invite friends qui renvoie vers une page permettant de déterminer ses proximités génotypiques avec d'autres usagers du site et de communiquer avec ces personnes via son profil Facebook enrichi d'informations génétiques personnelles. Nous avons alors affaire à une communauté fondée sur le partage d'informations génétiques qu’on peut appeler des biosocialités.

Le concept de biosocialité, initialement forgé par Paul Rabinow [21], repris ensuite [22], invite à dépasser l'opposition nature/culture et revêt deux significations conjointes: d'une part, il renvoie à une artificialité croissante de la nature à travers les découvertes et les applications biotechnologiques issues de la génomique; d'autre part, il permet de décrire et de qualifier l'apparition de nouvelles entités sociales (notamment des groupes ou associations de patients qui partagent une même séquence génétique) sous l'effet de ces dernières. Notons que les biosocialités émergeant autour des sites de vente directe de tests sont utilisées dans une optique commerciale, loin des expressions associatives auxquelles nous étions habitués jusqu'à maintenant: le partage de l'information génétique au sein de la communauté des utilisateurs d'un site et la possibilité de rencontrer des personnes sur les bases d'une similarité génétique sont présentés comme autant de services après vente associés à la présentation des tests proposés.

Ces biosocialités créées et suscitées par les entreprises posent de nombreuses questions, notamment anthropologiques: sont-elles susceptibles de conduire à un enrichissement des expériences et des identités humaines ou à une réduction de ces dernières au seul fait de partager une similarité génétique? Ce serait, dans ce dernier cas, oublier qu'elles tirent leur richesse de leur intrication au sein d'un vaste réseau de significations culturelles et sociales tout autant que biologiques [23].

\section{Conclusion}

Utilisation du mouvement santéiste, mise à profit des revendications contemporaines d'individualisation de la biopolitique et de curiosité pour les avancées de la génomique, et proposition de rencontres biosociales se présentent comme les trois principales stratégies commerciales mobilisées par les entreprises pourvoyeuses de tests génétiques sur Internet. Ces stratégies - dont il resterait à étudier l'efficacité réelle et à déterminer dans quelle mesure elle relèvent d'un calcul réel de la part de leurs promoteurs - présentent plusieurs enjeux. L'exploitation du santéisme est potentiellement anxiogène pour les usagers. La réutilisation des revendications contemporaines en faveur d'une biopolitique individualisée fournit un cadre d'expression pour ces dernières mais peut avoir pour effet de court-circuiter les modes traditionnels de certification scientifique et clinique au détriment de la qualité du service rendu aux usagers. Enfin, la politique de commercialisation des biocommunautés numériques, outre ses enjeux en termes de réduction de l'identitaire au génétique, risque d'avoir comme conséquence de priver certains groupes d'usagers des outils du débat démocratique. Celui-ci leur permettrait pourtant d'améliorer la qualité de ce service. Les blogs et forums mis à la disposition des usagers semblent être étroitement surveillés par les entreprises elles-mêmes, ne serait-ce que parce qu'elles publient des chartes de bonne conduite des prises de parole et ont recours à des dispositifs de flag post (qui permettent de signaler des abus dans les prises de parole) et à des community managers. Jusqu'à quel point les entreprises sont-elles prêtes à tolérer le développement d'un débat public et informé sur l'intérêt scientifique et clinique des tests qu'elles fournissent? L'intrication de ces communautés numériques avec la politique commerciale des sites est telle que l'on peut s'interroger sur les possibilités réelles de démocratie médicale et scientifique offertes par ces espaces de rencontre et de discussion. Sans doute y aurait-il là matière à une intervention des pouvoirs publics ou d'une autre instance qui viserait à développer sur Internet des espaces de discussion et d'information relatifs aux tests génétiques qui soient réellement au service des usagers et de leur opinion éclairée. $\diamond$

\section{SUMMARY}

Direct-to-consumer genetic testing through Internet: marketing, ethical and social issues

We probably did not anticipate all the consequences of the direct to consumer genetic tests on Internet, resulting from the combined skills of communication and genomic advances. What are the commercial strategies used by the companies offering direct-to-consumer genetic tests on Internet and what are the different social expectations on which they focus? Through a quantitative and qualitative analysis of the web sites offering such tests, it seems that these companies target a triple market based on: the "healthism" which raises health and hygiene to the top of the social values; the contemporary demands of the users to become 
actual actors of health decisions; and finally on the need for bio-social relationships. These three commercial strategies underlie various ethical and societal issues justifying a general analysis. $\diamond$

\section{REMERCIEMENTS}

Ce travail a été réalisé dans le cadre d'un contrat IRESP (appel à projets : services de santé - politiques publiques et santé 2009), et a reçu un soutien de la Commission européenne dans le cadre des contrats européens suivants $d u 7^{e}$ programme cadre : FP7/2007-2013, N²00754 GEN2PHEN, N² 23143 - TECHGENE et du projet PHGEN II, No 2008302 financé dans le programme de santé publique européen.

\section{CONFLIT D'INTÉRÊTS}

Les auteurs déclarent n'avoir aucun conflit d'intérêts concernant les données publiées dans cet article.

\section{RÉFÉRENCES}

1. Cambon-Thomsen A, Rial-Sebbag $\varepsilon$, Ducournau P. Tests génétiques : quels sont les enjeux du libre accès? Pour la Science $2009 ; 379: 24-7$.

2. Sénéchal K, Borry P, Howard HC, Avard D. Les tests génétiques offerts directement aux consommateurs : vue d'ensemble des normes et politiques applicables. GenEdit 2009; $7: 1-14$.

3. Hunter DJ, Khoury MJ, Drazen JM. Letting the genome out of the bottle - will we get our wish ? N Engl J Med $2008 ; 358: 105-7$.

4. Wallace H. Commercial influences and the story of personalised genomics. Communication présentée à la $5^{\text {th }}$ International DNA Sampling Conference, 16-18 septembre 2009, Banff, Canada. http://www.genomealberta.ca/APG/abstracts/wallace.aspx.

5. Hogarth S, Javitt G, Melzer D. The current landscape for direct-to-consumer genetic testing : legal, ethical and policy issues. Ann Rev Genomics Hum Genet 2008 ; 9: 161-82.

6. Janssens AC, Gwinn M, Bradley LA, et al. A critical appraisal of the scientific basis of commercial genomic profiles used to assess health risks and personalize health interventions. AmJ Hum Genet $2008 ; 82: 593-9$.

7. Hauser SL, Johnston SC. Personalized genetic scans : with gifts like these... Ann Neurol 2009 ; $65: 7-9$.

8. Howard HC, Borry P. Direct-to-consumer genetic testing: more questions than benefits ? Personalized Medicine $2008 ; 5: 317-20$.

9. Hennen L, Sauter A, Cruyce $\varepsilon$. Direct to consumer genetic testing European technology assesment group. Final report, $2008: 72 \mathrm{p}$.
10. Borry P, Sénéchal K, Avard D. Ethics watch: direct-to-consumer genome scanning services. Also for children? Nat Rev Genet 2009; $10: 8$.

11. Borry P, Sénéchal K, Avard D. Health-related direct-to-consumer genetic testing: a review of compagnies' policies with regard to genetic testing in minors. Fam Cancer $2010 ; 9: 51-9$.

12. Cassiman JJ. Introduction to over the counter genetic testing. Communication présentée au colloque organisé par le Conseil de l'Europe. Tests génétiques en accès libre et pharmacogénétique : quels enjeux individuels et collectifs en Europe? Paris : 2 octobre 2007.

13. Omran AR. The epidemiologic transition: a theory of the epidemiology of population change. Milbank Mem Fund 01971 ; 49 : 509.

14. Aïach P. La prévention, une idéologie de progrès? Agora Éthique Médecine Société 1994 ; 30 : 11-5.

15. Aïach P. Les voies de la médicalisation. In : Aïach $P$, Delanoë $D$, eds. L'ère de la médicalisation. Ecce homo sanitas. Paris : Éditions Economica, $1998: 15-35$

16. Sfez L. La santé parfaite. Critique d'une nouvelle utopie. Paris : Seuil, $1997: 398$ p.

17. Canguilhem G. Le normal et le pathologique. Paris: PUF, 1966: $224 \mathrm{p}$.

18. Ducournau P, Strand R. Trust, distrust and co-production: the relationship between research biobanks and donors. In : SolbakkJ H, Holm S, Hofmann B, eds. The ethics of research biobanking. Dordrecht: Springer Verlag, 2009 : 115-30.

19. Memmi D. Faire vivre et laisser mourir : le gouvernement contemporain de la naissance et de la mort. Paris : La Découverte, 2003: $310 \mathrm{p}$.

20. Fassin D, Memmi D. Le gouvernement des corps. Paris : Éditions de l'EHESS, $2004: 269 \mathrm{p}$.

21. Rabinow P. Artificiality and enlightenment: from sociobiology to biosociality. In: Essays on the Anthropology of Reason. Princeton: Princeton University Press, 1996: 91-112.

22. Gibbon S, Novas C. Biosocialities, genetics and the social sciences: making biologies and identities. Londres-New York : Routledge, $2008: 198 \mathrm{p}$.

23. Bibeau G. Le Québec transgénique. Science, marché, humanité. Montréal : Éditions du Boréal, 2004 : 454 p.

24. De Jager PL, Jia X, Wang J, et al. Meta-analysis of genome scans and replication identify CD6, IRF8 and TNFRSFIA as new multiple sclerosis susceptibility loci. Nat Genet $2009 ; 41: 776-82$.

25. Sagnes-Raffy C, Gourraud PA, Hannon V, et al. Multiple sclerosis in Haute-Garonne: an important underestimation of case numbers. Rev Epidemiol Sante Publ 2010 ; 58 : 23-31.

\section{TIRÉS À PART}

P. Ducournau

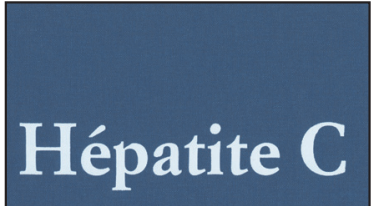

Jean-Michel Pawlotsky Daniel Dhumeaux

EDK

ISBN : 2-84254-096-4 512 pages

\section{Bon de commande}

À retourner à EDK, 2, rue Troyon - 92316 Sèvres Cedex

Tél. : 0155641393 - Fax : 0155641394 - E-mail : edk@edk.fr

NOM :

Prénom :

Adresse :

Code postal :

Ville :

Pays :

Fonction :

Je souhaite recevoir l'ouvrage Hépatite C : $65 €+3 €$ de port $=\mathbf{6 8} €$ TTC

en ................. exemplaire, soit un total de $\ldots$

$\square$ Par chèque, à l'ordre de $\mathbf{E} \mathbf{D} \mathbf{K}$

$\square$ Par carte bancaire : $\square$ Visa $\square$ Eurocard/Mastercard

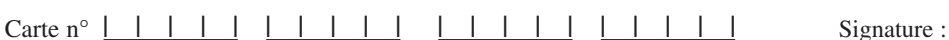

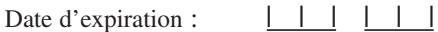

$\mathrm{N}^{\circ}$ de contrôle au dos de la carte : $\quad|\quad| \quad \mid$ 